\title{
THE IMPACT OF COGNITIVE FATIGUE ON AGE-RELATED DIFFERENCES IN NEUROMUSCULAR FUNCTION
}

\author{
A Thesis \\ by \\ ASHLEY E. SHORTZ \\ Submitted to the Office of Graduate and Professional Studies of \\ Texas A\&M University \\ in partial fulfillment of the requirements for the degree of \\ MASTERS OF SCIENCE IN PUBLIC HEALTH
}

$\begin{array}{ll}\begin{array}{ll}\text { Chair of Committee, } \\ \text { Committee Members, }\end{array} & \begin{array}{l}\text { Ranjana K Mehta } \\ \text { Ai Zhenens } \\ \text { Qi Zheng }\end{array} \\ \text { Head of Department, } & \text { Virender Sharma }\end{array}$

August 2015

Major Subject: Occupational Health

Copyright 2015 Ashley Shortz 


\section{ABSTRACT}

As the population of adults aged 65 and above is rapidly growing, it is crucial to identify physical and cognitive limitations pertaining to daily living. The aim of the study was to examine the impact of cognitive fatigue on age-related changes in neuromuscular fatigue and associated brain patterns of the prefrontal cortex.

12 younger (20-35 years) and 11 older (65 and above years) females performed intermittent handgrip exercises at 30\% maximum voluntary contraction (MVC) until voluntary exhaustion in the absence and presence of a prior 60-minute cognitively demanding task.

Cognitive fatigue was successfully and comparably induced across both the younger and older groups $(p<0.001)$. While neuromuscular fatigue outcomes (i.e., endurance time and rate of strength loss) and muscular responses were similar across groups (all $p>0.100$ ), greater decrements in cognitive fatiguerelated brain activation $(p=0.053)$ were observed in older adults when compared to younger adults.

Differences in brain activation suggest an age-related compensatory mechanism (i.e., the scaffolding theory of cognitive aging) of the frontal lobe to regulate the effect of cognitive fatigue to maintain neuromuscular performance. 


\section{ACKNOWLEDGEMENTS}

I would like to thank my committee chair, Dr. Ranjana Mehta, and my committee members, Dr. Adam Pickens, and Dr. Qi Zheng for their guidance and support throughout the course of this research.

Thanks also go to my friends and colleagues and the department faculty and staff for making my time while completing my Masters at Texas A\&M University a great experience. I also want to extend my gratitude to all the participants who were willing to offer their time to participate in the study.

Finally, thanks to my mother and father for their encouragement, support and love. 


\section{TABLE OF CONTENTS}

Page

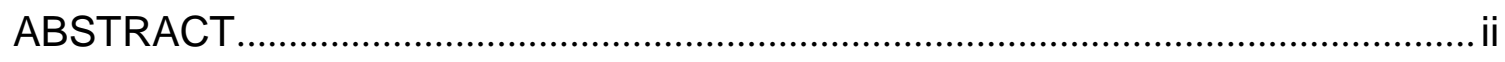

ACKNOWLEDGEMENTS ................................................................... ii

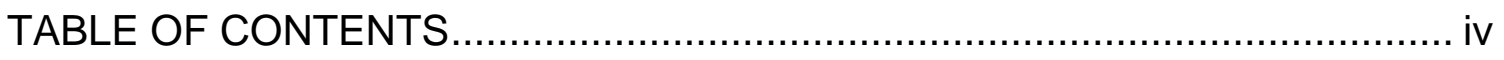

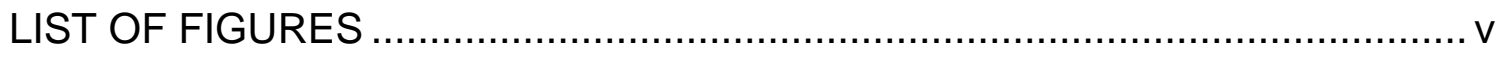

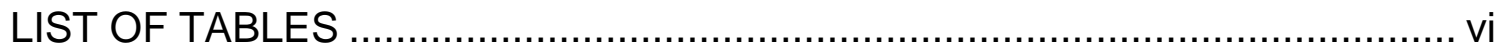

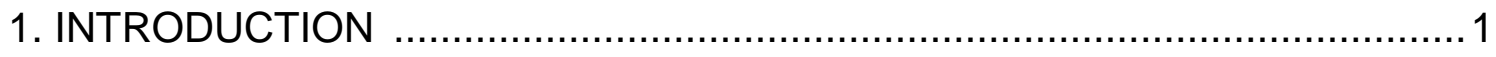

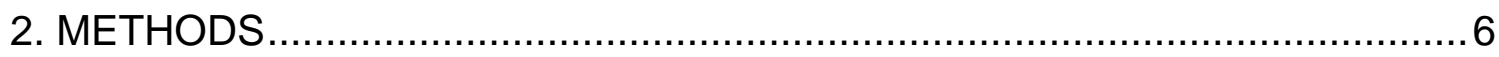

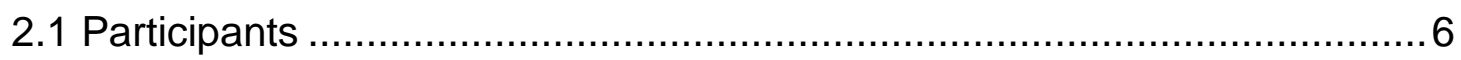

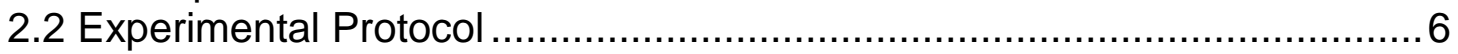

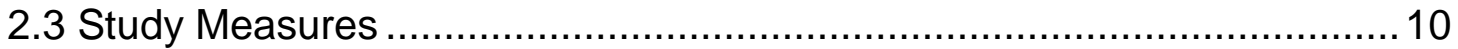

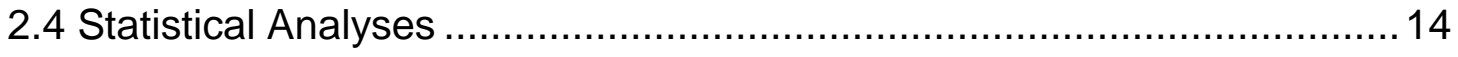

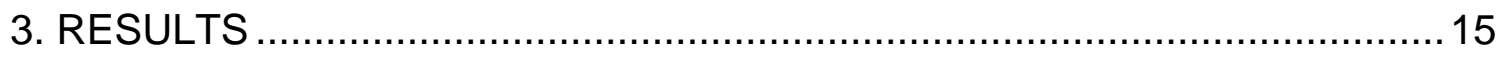

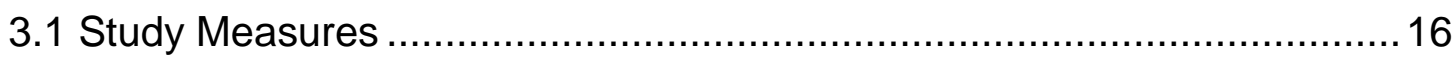

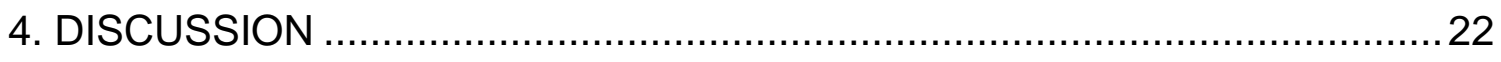

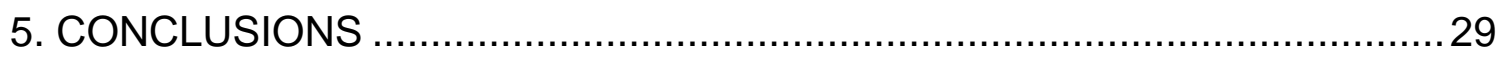

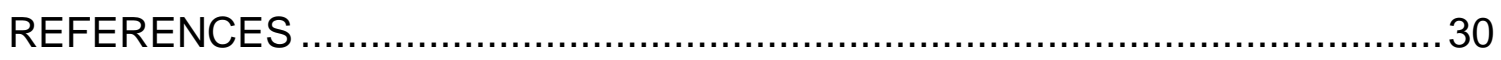




\section{LIST OF FIGURES}

Page

Figure 1: (A) Posture adopted during handgrip exercise with bioinstruments and (B) real-time feedback provided to participants. .....7

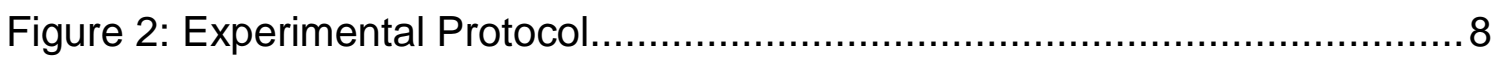

Figure 3: Protocol for cognitive fatigue (A) Stroop CW Test and (B) 1-Back. ......9

Figure 4. fNIRS probe placement according to the EEG 10-20 system. The solid black circles represent the sources and the black squares represent the detectors. The grey shaded area represents the brain region of interest.

Figure 5. POMS scores pre and post 60 minute Cognitive Fatigue. Error bars represent SE.

Figure 6: Percent change in (A) $\mathrm{HbT}$ and (B) $\mathrm{HbO}_{2}$ in the cognitive fatigue condition when compared to the control condition over time across both age groups in the left and right PFC. Error bars represent SE

Figure 7. Changes in (A) EMG RMS of ECR, (B) EMG RMS of FCR, (C) EMG $\mathrm{mF}$ of ECR, and (D) EMG $\mathrm{mF}$ of FCR over time across both age groups. Error bars represent SE.

Figure 8: Changes in force fluctuations over time across both age groups.

Error bars represent SE.

Figure 9: Changes in cardiovascular responses $(A)$ heart rate and $(B)$ heart rate variability over time across both age groups. Error bars represent SE. 


\section{LIST OF TABLES}

Page

Table 1. Summary Data of data for endurance time and rate of strength loss for two groups (mean (SD)) ..................................................... 16

Table 2. Summary data for RPD rate of change for two groups (mean

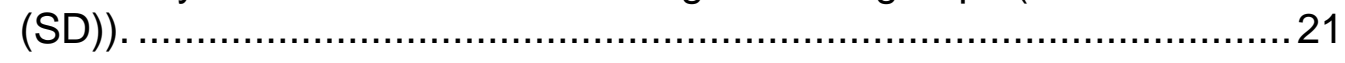




\section{INTRODUCTION}

Fatigue is a complex, multifaceted phenomenon that is associated with impairments in both physical and cognitive functioning and interference in performing daily tasks (Rantanen et al., 1999; Lorist, Boksem, \& Ridderinkhof, 2005; Power, Dalton, \& Rice, 2013). It refers to normal, everyday experiences that are observed after sustained physical activity or mental exertion (Smets, Garssen, Bonke, \& De Haes, 1995; Rantanen, et al., 1999; Cook, O'Connor, Lange, \& Steffener, 2007). Specific to physical functioning, neuromuscular fatigue can be defined as a reduced ability to generate a desired muscle force, which could be due to a number of factors including contractibility of muscle fibers, metabolic factors, or excitation rates of motorneurons (Allman \& Rice, 2002; Lorist et al., 2005). On the other hand, cognitive fatigue occurs due to prolonged periods of performing mentally demanding task that induces a state of subjective fatigue, feelings of "tiredness and lack of energy", and performance decrements (Lorist et al., 2005; Marcora, Staiano, \& Manning, 2009). Recent research elucidates the negative impact of cognitive fatigue on neuromuscular function, which includes changes in strength, muscle fatigue resistance, and joint steadiness (Marcora et al., 2009; Clark \& Taylor, 2011; Mehta \& Agnew, 2011, 2012, 2013; Shortz, Van Dyke, \& Mehta, 2012). Concomitant to these biomechanical outcomes, cognitive fatigue has shown to limit activation of the 
prefrontal cortex (PFC) during fatigue development (Shortz et al., 2012; Mehta \& Parasuraman, 2014).

It should be noted that the existing biomechanical and neuroimaging studies that investigate the impact of physical and cognitive fatigue have primarily focused on younger adults. A recent study investigated the impact of cognitive fatigue demand on biomechanical indicators of neuromuscular control in older adults and reported that older adults exhibited impaired joint steadiness of upper arm muscles due to imposed cognitive demand when compared to younger adults during a non-fatiguing task (Pereira et al., 2015). Because the normal aging process influences both physical and cognitive fatigue development processes, it is likely that the nature and extent to which cognitive fatigue affects neuromuscular fatigue development in older adults differs that from younger adults; however there are no published biomechanical or neuroimaging data. It is estimated that by 2030 , approximately $20 \%$ of the U.S. population will be composed of individuals aged 65 and above and is expected to double to 89 million by 2050 (CDC, 2013), therefore there is an urgent need to identify the impact of cognitive fatigue on age-related changes neuromuscular fatigue development and associated brain activation patterns. In the absence of such information, the promise of developing evidence-based therapeutic or preventive strategies for improving neuromuscular function in the elderly will likely remain limited. 
One of the vital functions related to functional independence and activities of daily living (ADL) is the ability to grasp and hold objects (Ranganatham, Siemionow, Sahgal, \& Yue, 2001). This ability requires a complex series of inputs from various body systems including the central nervous system (CNS) as well as the musculoskeletal system (Clark \& Taylor, 2011; Lin et al., 2014). With advancing age, there are normal structural and functional changes that occur in the brain and within the musculoskeletal system, which may impact hand functions and the ability to perform ADL such eating, mobility, and personal hygiene. Older adults exhibit general decrements in grasping/holding due to age-related reduction in grip strength (Lin et al., 2014). However, aging is associated with an increase in type I muscle fibers that has shown to increase fatigue resistance in older adults (Yoon, Schlinder, Griffith, \& Hunter, 2008; Avin \& Law, 2011). Age-related changes at the CNS, particularly the brain, include reduced cerebral blood flow, loss of cortical excitability, reduction in cortical plasticity, and loss of grey matter (Clark \& Taylor, 2011; Noble, Eng, Kokotilo, \& Boyd, 2011). Functional brain imaging studies have shown age-related increased compensatory activation at the PFC, ipsilateral cortical motor and sensorimotor areas to maintain motor performance (Noble et al., 2011; Boudrias et al., 2012). Conversely, reduced brain activity or inefficient cortical connectivity is observed in these brain regions, particularly the PFC, in older adults during fatiguing exercises (i.e. when task performance is not maintained) suggesting 
that PFC may be a limiting factor for impaired neuromuscular performance (Benwell, Bynes, Mastaglia, \& Thickbroom, 2005; Shibuya \& Kubnyama, 2007).

Cognitive fatigue, like physical fatigue, has shown to adversely impact numerous cognitive functions including attention, working memory and executive control, and this is particularly evident in older adults (Rypma \& D'Esposito, 2000; Cook, O'Connor, Lange, \& Steffener, 2007). Working memory function (i.e., the ability to process and store information) is largely regulated by the PFC and is typically one of the first functions to deteriorate with age (Baddeley, 1992; Insel, Morrow, Brewer, \& Figueredo, 2006; Wang et al., 2011; Klaassen et al., 2014). In older adults, working memory function is crucial cognitive function that along with reasoning, language, and learning abilities, enables performance of ADLs (Baddeley, 1992). At higher working memory loads, older adults are required to recruit additional resources in order to compensate for the age-related declines in working memory function, specifically with reduction in activation of dorsomedial prefrontal cortex (Klaassen et al., 2014). Moreover, older adults rely extensively on the PFC to maintain cognitive task performance (Cabeza, 2001; Cook et al., 2007; Klaassen et al., 2014).

Given that the PFC plays a major role in regulating both cognitive and neuromuscular performances, and that the normal aging process impacts PFC functioning, it is likely that age-related changes in PFC activity may moderate 
neuromuscular function when individuals are both cognitively and physically fatigued. However no published data exists to date. The purpose of this study was to examine the impact cognitive fatigue on age-related changes in neuromuscular fatigue and associated PFC activity. It is hypothesized that cognitive fatigue will influence neuromuscular fatigue development and this relationship will be associated with altered PFC patterns. Additionally, it is hypothesized that the normal aging process will influence these outcomes. 


\section{METHODS}

\subsection{Participants}

A total of 23 female adults participated in this study. Participants were divided into two groups: younger $(n=12)$ aged 20-35, with mean (SD) age, height, and body mass of $24(1.65)$ years, $1.60(0.12) \mathrm{m}$, and $65.69(13.01) \mathrm{kg}$, respectively and older ( $n=11$ ) aged 65 and above, with mean (SD) age, height, and body mass of $75.82(7.40)$ years, $1.59(0.11) \mathrm{m}$, and $78.65(15.59) \mathrm{kg}$, respectively. All participants were screened for right-handedness, color-blindness, sedentary lifestyles, and self-reported musculoskeletal injuries of the upper extremity currently or within the past year. Additionally, the older participants were screened for cognitive impairments using the Mini-Cog ${ }^{\mathrm{TM}}$ test (Borson, Scanlan, Chen, \& Ganguli, 2003). Written informed consent, using approved procedures by the institutional review board was obtained from all participants.

\subsection{Experimental Protocol}

A 2-Age (Younger vs Older) and 2-Cognitive Fatigue (Control vs Cognitive Fatigue) mixed effects model was employed to investigate the impact of cognitive fatigue on age-related changes in neuromuscular function and associated PFC activity. 
Participants attended two experimental sessions that were counterbalanced and separated by at least 48 hours to minimize order, learning, and fatigue effects. At the start of the study, participants were familiarized with the protocol and instructed to perform a series of warm-up handgrip exercises. After adequate warm-up, participants were bioinstrumented with various sensors described later. Once instrumented, a 2-minute functional baseline was collected where participants were instructed to focus on red mark placed within their seated eyelevel. After the functional baseline, a series of at least three maximum voluntary contractions (MVCs) were collected to obtain handgrip strength using a handgrip dynamometer (BIOPAC Systems, Inc., Santa Barbara, CA, USA) with sufficient rest periods of $\sim 2$ minutes between each collection, in a seated posture illustrated in Figure 1.

A

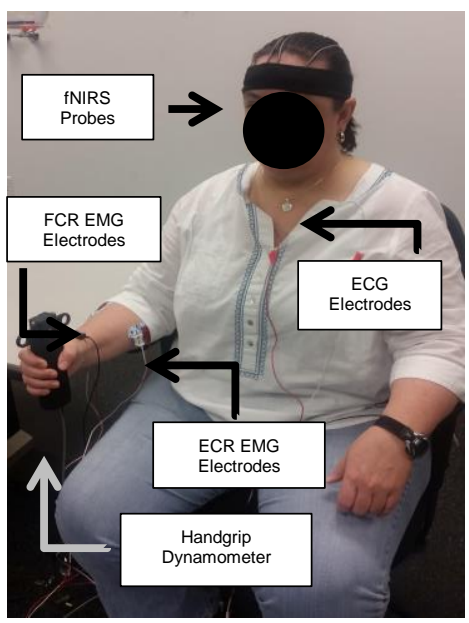

B

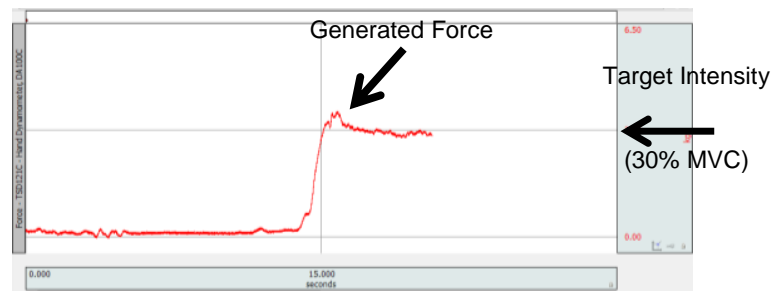

Figure 1: (A) Posture adopted during handgrip exercise with bioinstruments and (B) real-time feedback provided to participants. 
The experimental task required participants to intermittently perform handgrip exercises at $30 \%$ of maximal handgrip strength for 15 seconds (i.e., work cycle) with 15 seconds of rest between each gripping action until voluntary exhaustion, in the absence (control) and presence of cognitive fatigue (Figure 2).

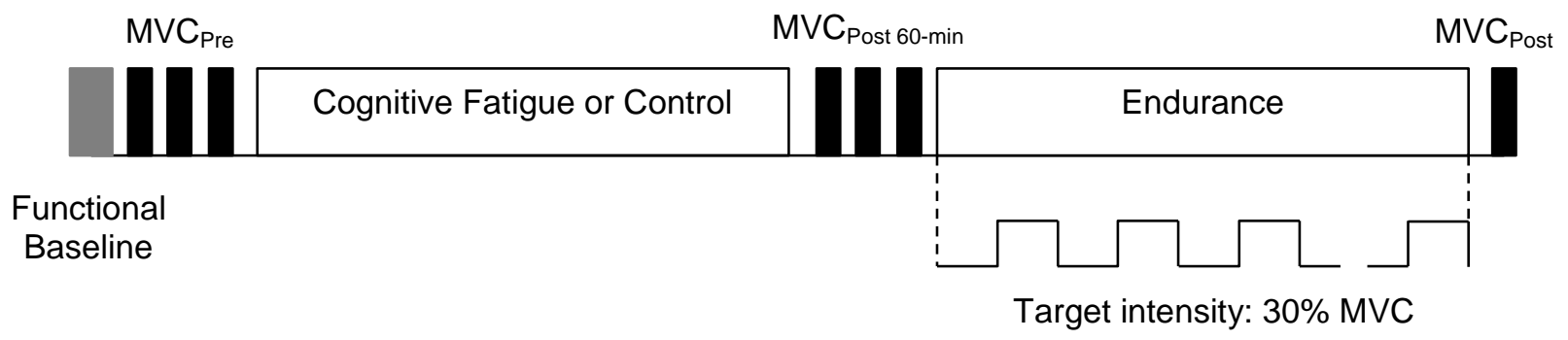

Figure 2: Experimental Protocol.

In the control condition, participants underwent a 60-minute quiet session, where they watched a documentary style DVD and/or read a magazine (i.e., National Geographic). The cognitive fatigue condition was composed of two tests of 30 minutes each: 1) Stroop Color Word (CW) test and 2) 1-Back test. The Stroop CW and 1-Back tasks were chosen to target basic working memory functions and have shown to induce cognitive fatigue when performed for a longer duration (Milham et al., 2002; Fairclough \& Houston, 2004; Qin, Hermans, van Marle, Luo, \& Fernandez, 2009; Klaassen et al., 2014). Adleman et al. (2002) has previously described the protocol for the Stroop CW test. The Stroop CW test was composed of 3 blocks: 1) color 2) work and 3) color-word (Figure 3A). 
A

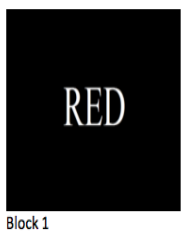

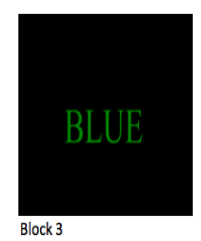

B

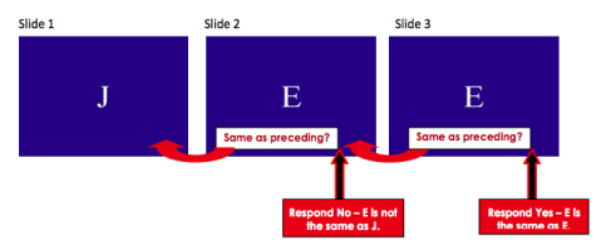

Figure 3: Protocol for cognitive fatigue (A) Stroop CW Test and (B) 1-Back.

During the 1-Back test, participants responded only when the letter that was currently on the display was the same as the preceding letter (Figure 3B). Throughout the 60-minute task, subjective reports were verbally obtained to verify that participants were cognitively fatigued. For the purpose of this study, cognitive fatigue was operationally defined as increase in self-reported Profile of Moods States (POMS) fatigue subscale score (Holtzer et al., 2011). The POMS fatigue subscale was used to quantify perceived fatigue (Marcora et al., 2009; Klaassen et al., 2014). The POMS fatigue subscale is composed of a series of 7 adjectives and is rated on a 0-4 scale (0- not at all to 4- extremely) for a total possible score of 28 . POMS scores were obtained twice: 1) pre 60-minute control or cognitive fatigue and 2) post 60-minute control or cognitive fatigue. 


\subsection{Study Measures}

\section{Neuromuscular Fatigue Outcomes}

Endurance time (ET) was determined as the time until voluntary exhaustion and was calculated when participants were unable to maintain the task intensity at $30 \% \pm 5 \% \mathrm{MVC}$ for at least 3 seconds. Rate of strength loss (i.e., [ $\mathrm{MVC}_{\text {pre }^{-}}$ $\left.M V C_{\text {post }} / M C_{\text {pre }} / \mathrm{ET}^{*} 100\right)$, regarded as the "gold standard" of muscle fatigue, was used to assess neuromuscular fatigue (Vøllestad, 1997), whereby an increase in the rate of strength loss is indicative of neuromuscular fatigue.

\section{PFC Activity}

PFC activity was measured using functional near infrared spectroscopy (fNIRS). fNIRS probes were placed bilaterally on the participants' forehead according to the international EEG 10-20 system over Fp1 on the left and Fp2 on the right (Figure 4).
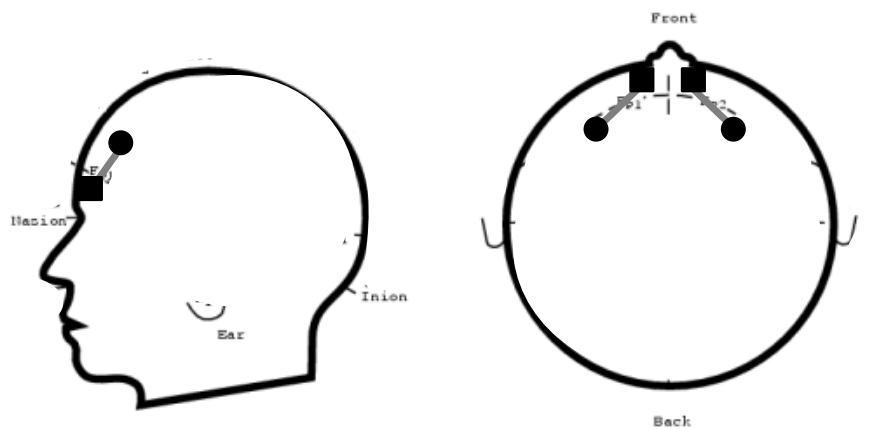

Figure 4. fNIRS probe placement according to the EEG 10-20 system. The solid black circles represent the sources and the black squares represent the detectors. The grey shaded area represents the brain region of interest. 
fNIRS data was collected using two fNIRS systems due to equipment mobility restrictions. The Hamamatsu system (NIRO 200 NX, Hamamatsu Photonics, Japan) was used when testing older participants due to the system being compact and portable which allowed for on-site collection in independent living facilities. The Techen CW6 (CW6 System, TechEn, Milford, MA) was used when testing younger participants in a laboratory setting. Changes in oxygenated $\left(\mathrm{HbO}_{2}\right)$ and total hemoglobin $(\mathrm{HbT})$ were recorded continuously from the two systems throughout each session. $\mathrm{HbO}_{2}$ was selected because it is the indicator with the greatest sensitivity for regional cerebral blood flow and indicative of neuronal activity when $\mathrm{HbT}$ is stable (Hoshi, Kobayashi, \& Tamura, 2001; Ayaz, Shewokis, Bunce, \& Izzetoglu, 2012). Maximum $\mathrm{HbO}_{2}$ and $\mathrm{HbT}$ levels for each work cycle was determined using the middle 10-second window and averaged across five stages normalized to each individual's endurance time, namely initial, $25^{\text {th }} \%, 50^{\text {th }} \%, 75^{\text {th }} \%$, and at exhaustion. In order to compare $\mathrm{HbO}_{2}$ and $\mathrm{HbT}$ values obtained from the two systems, percent $\mathrm{HbO}_{2}$ and $\mathrm{HbT}$ changes $\left(\% \Delta \mathrm{HbO}_{2}, \% \Delta \mathrm{HbT}\right)$ were computed between the control and cognitive fatigue conditions $\left(\left(\mathrm{HbO}_{2_{\text {cognitive fatigue }}}-\mathrm{HbO}_{2_{\text {control }}}\right)^{*} 100 / \mathrm{HbO}_{2_{\text {control }}}\right)$ within each age group. 


\section{Muscular Responses}

Surface electromyography (EMG) was used to examine muscle fatigue development. Surface EMG electrodes (BIOPAC Systems, Inc., Santa Barbara, CA, USA) were placed on the lower arm muscles (extensor carpi radialis (ECR) and the flexor carpi radialis (FCR)). These muscles were chosen as they have shown to be activated during handgrip actions (Hoozemans \& van Dieën, 2005; Finneran \& O'Sullivan, 2013). The muscle sites were shaved and cleansed to reduce skin resistance prior to placement surface EMG electrodes. Surface EMG electrodes were placed along the belly of the ECR and FCR in the direction of the muscle fiber. All EMG signals were collected at $1000 \mathrm{~Hz}$ and recorded continuously (Biopac Systems, Santa Barbara, CA, USA). EMG amplitude (EMG RMS) and mean frequency (EMG mF) values for each muscle site were averaged across the middle 10 -seconds for each stage (initial, $25^{\text {th }} \%$, $50^{\text {th }} \%, 75^{\text {th }} \%$, and at exhaustion) and evaluated to assess differences between conditions. An increase in EMG RMS and EMG $\mathrm{mF}$ over time has shown to be strong indicators of muscular fatigue (Madeleine, Bajaj, Sogaard, \& ArendtNielsen, 2001; Cifrek, Medved, Tonkovic, \& Ostojic, 2009).

\section{Force Fluctuations}

Force fluctuations were measured (i.e., standard deviation/mean force * 100) during the fatiguing handgrip exercises using force output from the handgrip dynamometer. Data was collected at $1000 \mathrm{~Hz}$ and recorded continuously during 
the duration of the task. The middle 10-second window of the work cycle was averaged across five stages (initial, $25^{\text {th }} \%, 50^{\text {th }} \%, 75^{\text {th }} \%$, and at exhaustion). An increase in the force fluctuations has shown to be associated with fatigue progression (Contessa, Adam, \& De Luca, 2009).

\section{Cardiovascular Responses}

Cardiovascular measures included heart rate $(\mathrm{HR})$ and heart rate variability (HRV). A 3-electrode electrocardiogram based on the III-lead configuration was used to obtain HR and HRV low/high frequency ratio (LF/HF). Data was collected at $1000 \mathrm{~Hz}$ and continuously throughout each condition. HR was recorded as beat-to-beat $(R-R)$ intervals and average across the middle 10second window of the work cycle was averaged across five stages (initial, $25^{\text {th }} \%$, $50^{\text {th }} \%, 75^{\text {th }} \%$, and at exhaustion). An increase in HR and an increase in HRV LF/HF are indicative of cognitive stress (Thayer, Ahs, Fredrikson, Sollers III, \& Wager, 2012).

\section{Subjective Reports}

The Borg CR-10 scale (Borg, 1990) was used to collect Ratings of Perceived Discomfort (RPDs) on a 0-10 scale (0- not at all to 10- extreme discomfort). Participants were asked to report RPD every two minutes during the fatiguing exercise, and the rate of change for RPD was obtained. An increase in RPD rate is indicative of increased discomfort due to fatigue (Mehta, In press) 


\subsection{Statistical Analyses}

In order to verify that the Stroop CW and 1-Back tests successfully induced cognitive fatigue, a three-way analysis of variance (ANOVA) was performed to determine the effects of age (Younger vs. Older), cognitive fatigue (Control vs. Cognitive Fatigue) and time (Pre $\mathrm{CF}_{\mathrm{C}}$ vs. Post $\mathrm{CF}_{\mathrm{C}}$ ) on POMS scores. A two-way ANOVA was performed to determine the effects of age (Younger vs. Older), cognitive fatigue (Control vs. Cognitive Fatigue) on endurance time and rate of strength loss. A 3-way ANOVA was completed to determine the effects of age (Younger vs. Older), hemisphere (Right vs. Left), and time (5 time stages) on $\% \Delta \mathrm{HbO}_{2}$ and $\% \Delta \mathrm{HbT}$. Additionally, separate ANOVAs were conducted to determine the effects of age (Younger vs. Older), cognitive fatigue (Control vs. Cognitive Fatigue), and time (5 time stages) on EMG RMS, EMG mF, force fluctuations, HR, and HRV. Lastly a 2-way ANOVA was performed to determine the effects of age (Younger vs. Older) and cognitive fatigue (Control vs. Cognitive Fatigue) on RPD scores. Statistical significance was determined when $\alpha<0.05$. All statistical analyses were conducted using SPSS 22 (IBM SPSS Statistics, IBM Corp., Armonk, NY, USA). 


\section{RESULTS}

POMS scores increased significantly over time $\left(F=38.625, p<0.001, \eta_{p}{ }^{2}=0.648\right)$ and after the 60-minute CF condition when compared to the control condition $\left(F=11.257, p=0.003, \eta_{p}^{2}=0.349 ;\right.$ Figure 5). However, the interaction between cognitive fatigue $\times$ time $\left(F=30.215, p<0.001, \eta_{p}^{2}=0.590\right)$ indicated that the increase in POMS score over time was only observed in the cognitive fatigue condition. Similar scores were observed across younger and older adults $(p=0.605)$, implying that cognitive fatigue was successfully manipulated and was comparable across both age groups.

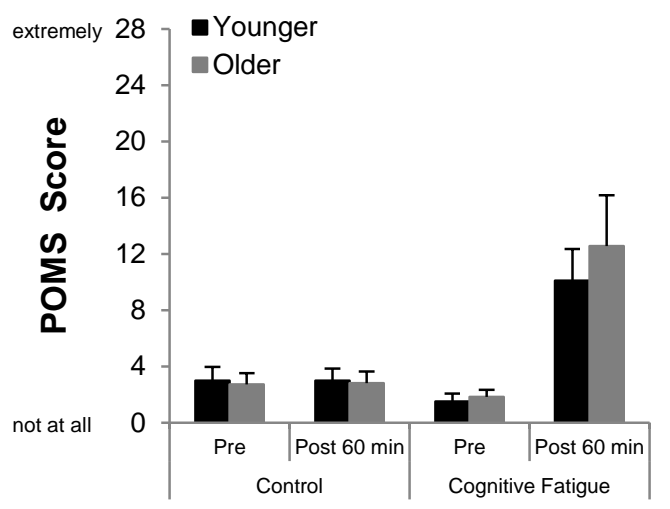

Figure 5. POMS scores pre and post 60 minute Cognitive Fatigue. Error bars represent SE. 


\subsection{Study Measures}

\section{Neuromuscular Fatigue Outcomes}

Endurance times and rate of strength loss for the younger and older groups are summarized in Table 1. Endurance times and rate of strength loss were similar across both age groups and cognitive fatigue conditions (all $p>0.100$ ).

Additionally, there was no interaction effect of age $\times$ cognitive fatigue on either of these neuromuscular fatigue outcomes (both $p>0.125$ ).

Table 1. Summary Data of data for endurance time and rate of strength loss for two groups (mean (SD)).

\begin{tabular}{|c|c|c|c|c|}
\hline \multirow{2}{*}{ Measure } & \multicolumn{2}{|c|}{ Younger $(\mathrm{n}=12)$} & \multicolumn{2}{c|}{ Older $(\mathrm{n}=11)$} \\
\cline { 2 - 5 } & Control & $\begin{array}{c}\text { Cognitive } \\
\text { Fatigue }\end{array}$ & Control & $\begin{array}{c}\text { Cognitive } \\
\text { Fatigue }\end{array}$ \\
\hline $\begin{array}{c}\text { Endurance } \\
\text { Time (min) }\end{array}$ & $26.25(16.27)$ & $25.29(26.74)$ & $24.42(21.06)$ & $21.75(17.79)$ \\
\hline $\begin{array}{c}\text { Rate of } \\
\begin{array}{c}\text { Strength Loss } \\
\text { (\%MVC/min) }\end{array}\end{array}$ & $2.59(2.29)$ & $5.14(5.50)$ & $2.36(2.92)$ & $2.38(2.43)$ \\
\hline
\end{tabular}

PFC Activity

$\% \triangle \mathrm{HbT}$, across the right and left PFC hemispheres, were not significantly affected by age, task, and time or the interaction between age and task (all $p$ $>0.114$; Figure 6). However, the interaction of time and hemisphere was found significant $\left(F=2.630, p=0.050, \eta_{p}{ }^{2}=0.226\right)$, along with their interaction with age $\left(F=3.196, p=0.024, \eta_{p}^{2}=0.262\right)$. Post hocs indicated that $\% \Delta H b T$ were significantly different between the hemispheres at the initial stage; however this 
was only observed in younger adults. Since $\% \Delta \mathrm{HbT}$ remained stable over time and across tasks and age groups independently, further analyses of brain activity were performed using $\mathrm{HbO}_{2}$. There were no main effects of time $(p=0.324)$ and hemisphere $(p=0.596)$ on $\% \Delta \mathrm{HbO}_{2}$. However, a marginal effect of age was observed $(p=0.053)$ with greater decrements in $\% \Delta \mathrm{HbO}_{2}$ (i.e., during the cognitive fatigue when compared to the control condition) in the older group, while $\% \Delta \mathrm{HbO}_{2}$ in the younger group were similar across the two cognitive fatigue conditions. No significant differences in $\% \Delta \mathrm{HbO}_{2}$ for any two- or threeway interactions between age, task, or time were observed (all $p>0.222$ ).

A

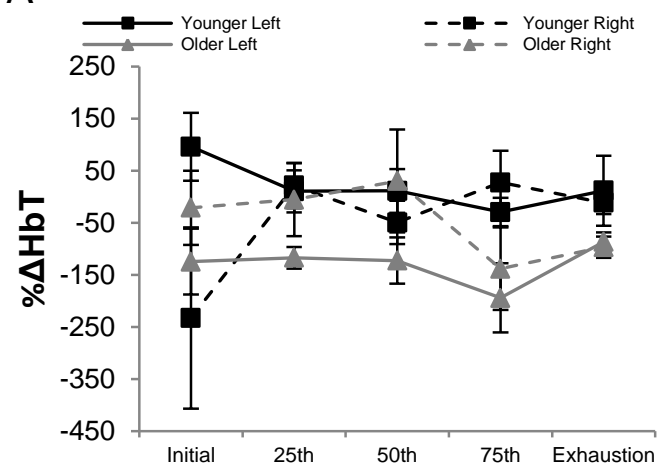

B

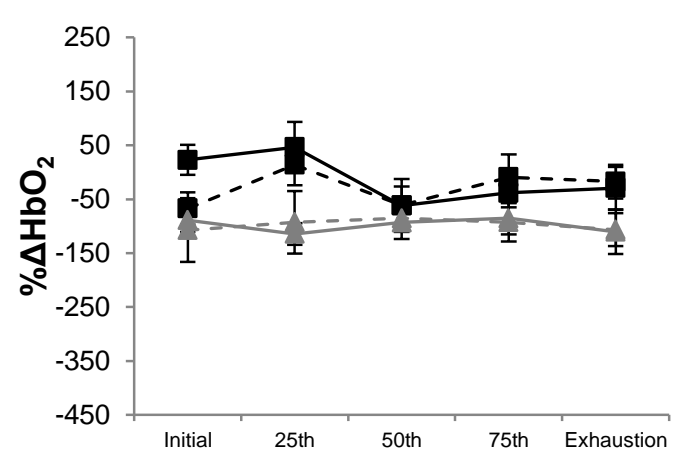

Figure 6: Percent change in (A) $\mathrm{HbT}$ and (B) $\mathrm{HbO}_{2}$ in the cognitive fatigue condition when compared to the control condition over time across both age groups in the left and right PFC. Error bars represent SE.

\section{Muscular Responses}

EMG RMS of the ECR and FCR muscles both significantly increased over time (ECR: $F=4.054, p=0.005, \eta_{p}^{2}=0.162$, FCR: $F=2.588, p=0.043, \eta_{p}^{2}=0.110$; Figure 
5). Post hoc comparisons using Bonferroni correction revealed that EMG RMS for the ECR was significant at exhaustion compared to the $50^{\text {th }}$ stage. However, post hoc comparisons for FCR revealed no significant effects between the different time stages. There were no main effects observed for age and task, or their interactions, on EMG RMS for both muscles (all p>0.235). Similarly, EMG $\mathrm{mF}$ for both ECR and FCR muscles were not influenced by age, task, or their interaction (all p>0.189; Figure 6).

A

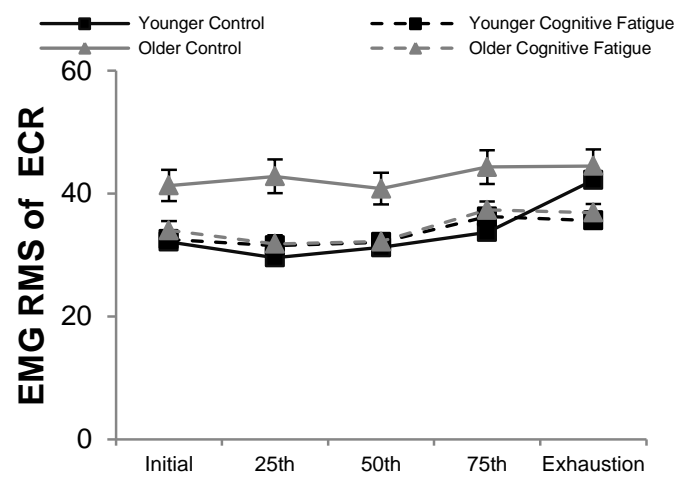

C

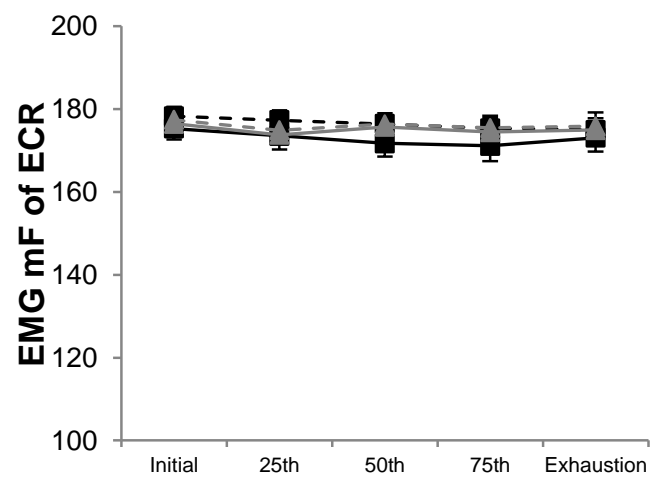

B

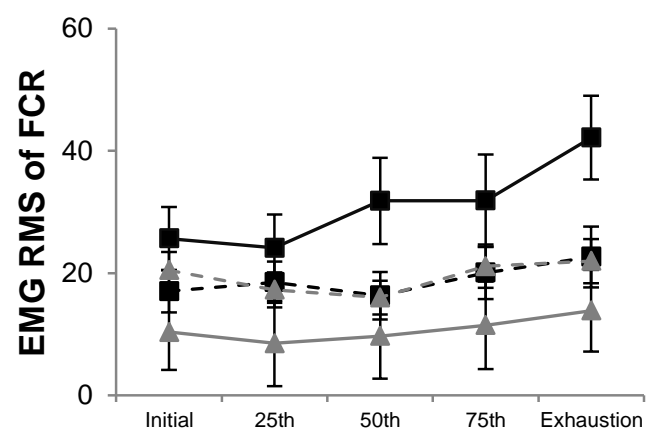

D

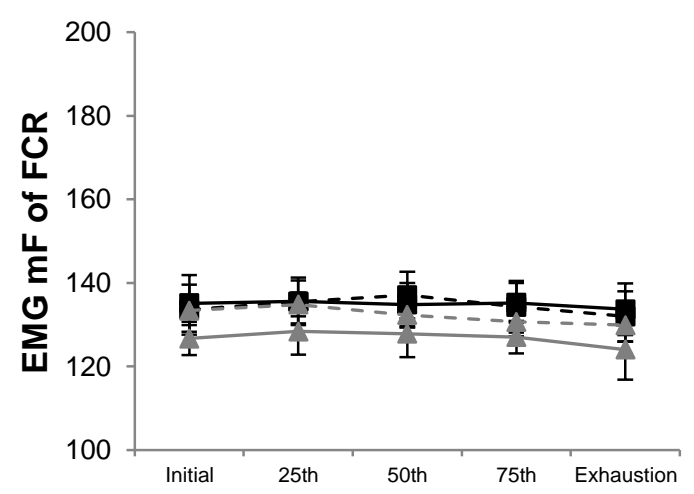

Figure 7. Changes in (A) EMG RMS of ECR, (B) EMG RMS of FCR, (C) EMG $\mathrm{mF}$ of ECR, and (D) EMG $\mathrm{mF}$ of FCR over time across both age groups. Error bars represent SE. 


\section{Force Fluctuations}

No significant effects of age $(p=0.128)$, cognitive fatigue $(p=0.267)$ on force fluctuations were observed. A marginal effect of time was observed $(p=0.073)$, with greater fluctuations observed at exhaustion when compared to the $50^{\text {th }}$ stage $(p=0.003)$. Additionally, there was a three-way interaction between age $\times$ cognitive fatigue $\times$ time $\left(F=2.701, p=0.036, \eta_{p}^{2}=0.114\right)$; greater fluctuations were observed in the younger group when compared to the older group during the cognitive fatigue condition at the $75^{\text {th }}$ time stage.

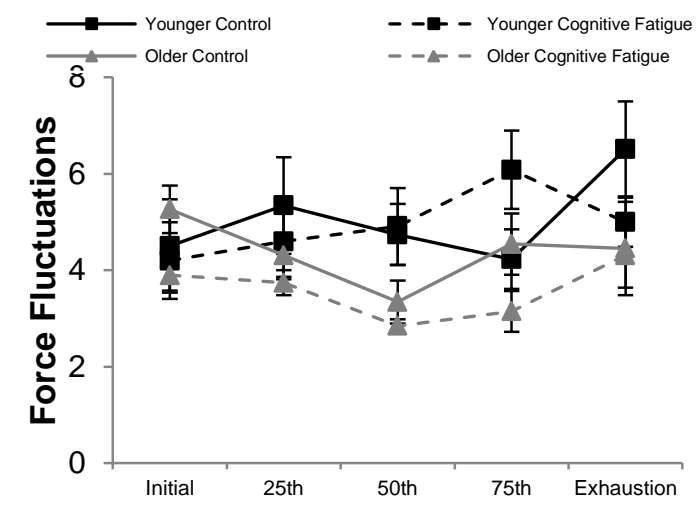

Figure 8: Changes in force fluctuations over time across both age groups. Error bars represent SE.

\section{Cardiovascular Responses}

While HR increased linearly over time $\left(F=3.883, p=0.006, \eta_{p}{ }^{2}=0.170\right.$; Figure 9). it was similar across both the control and cognitive fatigue conditions $(p=0.169$; 
Figure 9) and across both age groups ( $p=0.501)$. Additionally, no two-way interactions between age, task, and time were observed (all $p>0.139$ ). However there was a three-way interaction between age $\times$ cognitive fatigue $\times$ time $\left(F=2.491, p=0.050, \eta_{p}^{2}=0.116\right)$; greater HR was observed in the older group when compared to the younger group during the control condition at the $75^{\text {th }}$ time stage. Similar to HR, HRV LF/HF was consistent for both conditions $(p=0.392)$ and age $(p=0.437)$. However, there was an interaction between cognitive fatigue $\times$ age $\left(F=8.089, p=0.010, \eta_{p}^{2}=0.278\right)$ on the $H R V L F / H F$, with a higher ratio observed in the cognitive fatigue condition (when compared to control) in younger adults. However no differences were observed in the older age group. No other interaction effects were observed $(p>0.154)$.

A

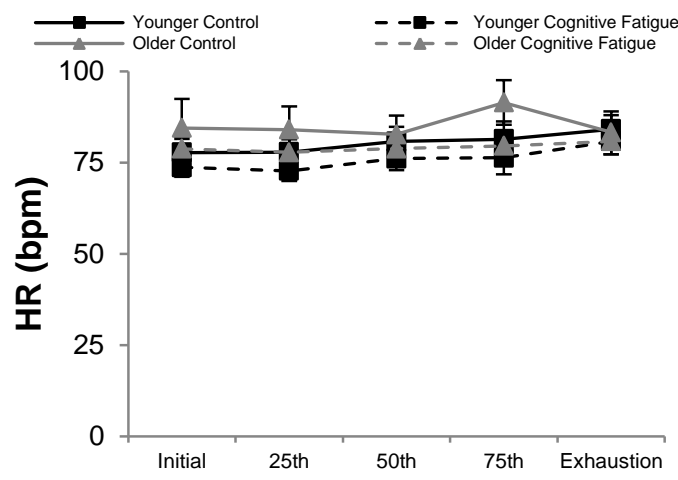

B

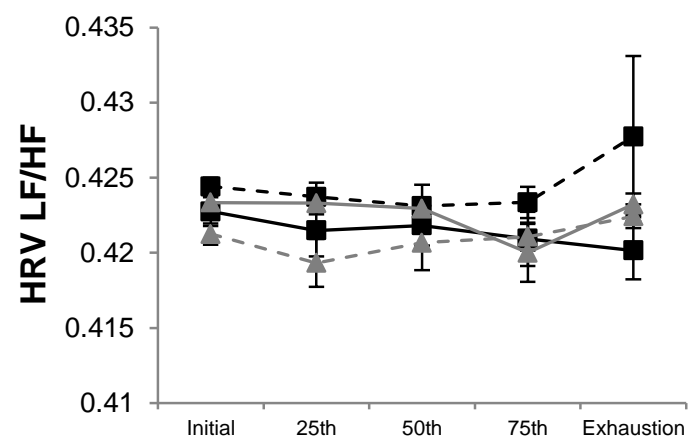

Figure 9: Changes in cardiovascular responses $(A)$ heart rate and $(B)$ heart rate variability over time across both age groups. Error bars represent SE. 


\section{Subjective Reports}

Rate of change for RPD was similar between age $(p=0.816)$ and cognitive fatigue $(p=0.798 ;$ Table 2$)$. No interaction effect for age $\times$ task $(p=0.624)$ was observed.

Table 2. Summary data for RPD rate of change for two groups (mean (SD)).

\begin{tabular}{|c|c|c|c|c|}
\hline \multirow{2}{*}{ Measure } & \multicolumn{2}{|c|}{ Younger $(\mathrm{n}=12)$} & \multicolumn{2}{c|}{ Older $(\mathrm{n}=11)$} \\
\cline { 2 - 5 } & Control & $\begin{array}{c}\text { Cognitive } \\
\text { Fatigue }\end{array}$ & Control & $\begin{array}{c}\text { Cognitive } \\
\text { Fatigue }\end{array}$ \\
\hline $\begin{array}{c}\text { RPD rate of } \\
\text { change }(/ \mathrm{min})\end{array}$ & $0.757(0.15)$ & $0.749(0.20)$ & $0.922(0.40)$ & $0.960(0.29)$ \\
\hline
\end{tabular}




\section{DISCUSSION}

The present study investigated the impact of cognitive fatigue on age-related changes in neuromuscular function and associated brain activation patterns of the PFC during submaximal fatiguing handgrip exercises. The results indicated that while cognitive fatigue did not impair neuromuscular functioning with aging, the normal aging process was associated with greater decrements in $\mathrm{HbO}_{2}$ levels in the PFC during the cognitive fatigue condition when compared to the control condition.

Neuromuscular capacity, measured as a function of endurance time, has shown to be negatively affected by cognitive stress prior to (Marcora et al., 2009) and during physical fatigue exercises (Mehta \& Agnew, 2012), specifically in younger adults. In the current study, prior cognitively fatigued state did not affect endurance time or rate of strength loss for both age groups. Methodological differences, such as duration of the prior cognitively fatigue task or the nature of physical fatigue exercise, may influence the findings. Marcora et al. (2009) investigated the effects whole body fatigue using a cycling fatigue protocol after completing a 90-minute continuous performance task, while the current study focused on localized muscle fatigue of the lower arm after a 60 -minute exposure to two working memory tasks. Moreover, previous studies that have investigated the impact of cognitive fatigue on muscle capacity have focused on postural (shoulder) and lower extremity (quadriceps) muscles rather than smaller muscle 
groups employed during handgrip exercises. Thus it is likely that the impact of cognitive fatigue on neuromuscular capacity is task- and muscle-dependent, as previously reported by Mehta et al. (2013). Since endurance time was not affected by cognitive fatigue for either age group, neuromuscular fatigue development (i.e., force fluctuations, EMG RMS, and EMG mF) was comparable across both conditions and age groups. While there were no age-related changes observed in neuromuscular capacity in the current study, research relating to fatigue resistance with aging remains unresolved (Yassierli, Nussbaum, Iridiastadi, \& Wojcik, 2007). A recent study demonstrated that high levels of cognitive demand increases force fluctuations of the lower arm in older adults (Pereira et al., 2015). It was suggested that the increase in fluctuations in the older adults may have been amplified due to age-related changes in the motorneuron pool via excitation or decreased inhibition when increased cognitive demand is present (Pereira et al., 2015).

Age-related reductions in strength and cognitive functions may predispose older adults to the effects of fatigue, especially when exposed to cognitive demand (Pereira et al., 2015). In the current study, cognitive fatigue was successfully induced using a 60-minute cognitively demanding task (i.e., 30-minute Stroop CW test and 30-minute 1-Back task) evidenced by the significant increase in POMS scores in both groups. Findings from this study suggest that older adults exhibited greater decrements in $\mathrm{HbO}_{2}$ levels as a result of cognitive fatigue 
when compared to their younger counterparts even though neuromuscular fatigue outcomes were similar (i.e., endurance time and rate of strength loss). While not significant, the younger group initially exhibited greater activation compared to exhaustion due to cognitive fatigue (Figure 6B). This trend could be explained by the initial need for additional resources placed by the cognitive demand in order to maintain task performance, as seen in previous research (Shortz et al., 2012). As this trend was not observed in the older group (relative stable $\sim 10.81 \%$ decrease in $\% \Delta \mathrm{HbO}_{2}$ over time; Figure $\left.6 \mathrm{~B}\right)$, it is possible that aging may differentially impact PFC regulation when individuals are both cognitively and physically fatigued. In support of this, previous research has demonstrated that that age-related reductions in PFC activation is observed during physically fatiguing exercises (Benwell et al., 2005; Shibuya \& Kubnyama, 2007) as well as during tasks involving the working memory (Cabeza, 2001). The age-specific difference in brain activation patterns of the PFC observed here may be indicative of compensatory mechanism(s) for neural degradation often seen with the normal aging process. In order to maintain task performance older adults through reorganization of cognitive function, which is commonly referred to as the scaffolding theory of cognitive aging. (Cabeza, Anderson, Locantore, \& McIntosh, 2002; Huang, Polk, Goh, \& Park, 2012; Park \& Reuter-Lornez, 2009; Cooper, Worthy, Gorlick, \& Maddox, 2013). This theory suggests that with aging there is a compensatory shift in neural recruitment to accommodate cognitive challenge (Park \& Reuter-Lornez, 2009; Cooper et al., 
2013). While the present study focused solely on the activation of the PFC, other brain regions are involved in fatigue development, particularly in older adults (Liu et al., 2003; Clark \& Taylor, 2011; Noble et al., 2011). Previous research has demonstrated a shift in activation centers in the brain to maintain neuromuscular performance during a fatiguing protocol (Liu et al., 2007). It is likely that the presence of cognitive fatigue accelerated cortical redistribution in older adults. Because only the PFC was monitored in the present study, further investigation is warranted to provide additional information about the proposed theory on shifting of brain activation centers when individuals are both physically and cognitively fatigued. The premotor and motor areas may be of interest due to their interconnection with the PFC during motor tasks under stress (Takahara et al., 2012). Particularly with aging, the PFC decreases in size which may alter connection to the premotor cortex and result in a decline in motor capabilities (Raz et al., 2005).

In general, heart rate increased linearly during neuromuscular fatigue development across both age groups, implying that all participants reached similar physiological fatigued states across both cognitive fatigue conditions (Thayer et al., 2012). This physiological increase over time was accompanied with an increased perception of discomfort. While heart rate and ratings of perceived exertions are highly correlated (Borg, Hassmen, \& Lagerstrom, 1987), these outcomes were not found sensitive to the different cognitive fatigue 
conditions, which is a departure from findings reported by Marcora et al. (Marcora et al., 2009). Marcora et al. (2009) demonstrated that a prior cognitive fatigued state is associated with greater perception of effort and discomfort and decreased muscle endurance. It is likely that the exposure to the cognitively fatiguing task may have played a role in the discrepancies observed between the two studies. Heart rate variability, as measured by the LF/HF ratio, was also found to be similar across both cognitive fatigue conditions and between the two age groups. While younger adults exhibited greater LF/HF ratio in the cognitive fatigue condition when compared to older adults, this increase was marginal ( $0.73 \%$ increase). Previous studies have reported stress-related changes in $\mathrm{HRV}$ indices (both temporal and spectral) during neuromuscular fatigue development (Mehta, Agnew, \& Nussbaum, 2013; Mehta, In press). However, current research is mixed regarding the effects of age-related changes in HRV indices due to cognitive fatigue (Pagani et al., 1991; R. Wood, W. Wood, Welsch, \& Avenal, 1998; R. Wood, Maraj, Lee, \& Reyes, 2002).

There are a few limitations in the present study that warrant discussion. First, the study examined the impact of cognitive fatigue on neuromuscular function and associated brain activity in younger and older females. This was done to avoid sex-specific differences in both cognitive and neuromuscular capacity. Pereira et al. (Pereira et al., 2015) reported that older females are more susceptible to the effects of neuromuscular fatigue; especially at high and low levels of cognitive 
demand during sustained upper arm exertions, when compared with males of the same age. Additionally, younger women exhibit greater fatigability, at higher levels of cognitive demand, compared to their male counterparts (Pereira et al., 2015). These sex differences may have important implications for work-related tasks that require either high or low levels of cognitive workload, particularly with the aging workforce. Future research is warranted to extent this investigation to include a larger sample of both males and females. Second, the present study monitored the PFC regions due to equipment constraints. Existing neuroimaging investigations of fatigue development suggest a shift in activation centers in the brain to compensate for fatigue-related loss in neural efficiency, particularly with the normal aging process (Liu et al., 2007; Clark \& Taylor, 2011; Noble et al., 2011), future research should examine activation of motor function-related brain regions to understand age-related changes in functional brain activation patterns when individuals are physically and cognitively fatigued. Third, while all participants reported that the 60-minute working memory tasks were cognitively fatiguing, it is possible that longer exposure to such stressors may result in greater negative effects of cognitive fatigue on neuromuscular function. Moreover, it is likely that such high exposures might have a greater impact on older adults. 
The findings have important implications for daily tasks involving some levels of cognitive demand and physical exercise, especially when combined. Additionally, the altered brain patterns of the PFC suggest different compensatory mechanisms with aging in order to regulate the effects of cognitive fatigue on neuromuscular fatigue. The current findings suggest that promoting brain health in healthy aging interventions may help to improve the quality of life and physical capabilities in older adults through enhancing neural networks of the aging brain. 


\section{CONCLUSIONS}

The purpose of this study was to evaluate the impact of cognitive fatigue on agerelated differences in neuromuscular function. The results indicate that while muscular responses were similar between conditions for both age groups, brain activation patterns in the PFC were different, with decrements in $\mathrm{HbO}_{2}$ levels in the older group, specifically in the cognitive fatigue condition. The difference in brain activation patterns could indicate an age-related compensatory mechanism of the PFC to regulate the impact of cognitive fatigue on neuromuscular function. The interaction of physical fatigue and cognitive fatigue may have important implications specifically for older adults. As older adults are more susceptible to the effects of fatigue, this interaction may have an impact on performing ADL, which may result in a loss of independence. 


\section{REFERENCES}

Adleman, N., Menon, V., Blasey, C., White, C., Warsofsky, I., Glover, G., et al. (2002). A developmental fMRI study of the stroop color-word task. Neurolmage , 16, 61-75.

Allman, B., \& Rice, C. (2002). Neuromuscular fatigue and aging: central and peripheral factors. Muscle \& Nerve , 25, 785-796.

Avin, K., \& Law, F. (2011). Age-related differences in muscle fatigue vary by contraction type: a meta-analysis. Phys Ther , 91, 1153-1165.

Ayaz, H., Shewokis, P., Bunce, S., \& Izzetoglu, K. (2012). Optical brain monitoring for operator training and mental workload assessment. Neurolmage , 59, 36-47.

Baddeley, A. (1992). Working Memory. Science , 255 (5044), 556-559.

Benwell, N., Bynes, M., Mastaglia, F., \& Thickbroom, G. (2005). Primary sensorimotor cortex activation with task-performance after fatiguing hand exercise. Exp Brain Res, 167 (160-164).

Borg, G. (1990). Psychophysical scaling with applications in physical work and the perception of exertion. Scand. J. Work. Environ. Health , 16, 55-58.

Borg, G., Hassmen, P., \& Lagerstrom, M. (1987). Perceived exertion related to heart rate and blood lactate during arm and leg exercises. European journal of applied physiology and occupational physiology , 56 (6), 679685. 
Borson, S., Scanlan, J., Chen, P., \& Ganguli, M. (2003). The Mini- Cog as a screen for dementia: validation in a population- based sample. Journal of the American Geriatrics Society , 51 (10), 1451-1454.

Boudrias, M.-H., Gonçalves, C., Penny, W., Park, C.-h., Rossiter, H., Talelli, P., et al. (2012). Age-related changes in causal interactions between cortical motor regions during hand grip. Neurolmage , 59, 3398-3405.

Cabeza, R. (2001). Cognitive neuroscience of aging: contributions of functional neuroimaging. Scand. J.of Psychology , 42, 277-286.

Cabeza, R., Anderson, N., Locantore, J., \& McIntosh, A. (2002). Aging

Gracefully: Compensatory Brain Actvity in High-Performing Older Adults. Neurolmage , 17, 1394-1402.

CDC. (2013). The State of Aging \& Health in America 2013. US Dept of Health and Human Services, Atlanta, GA.

Cifrek, M., Medved, V., Tonkovic, S., \& Ostojic, S. (2009). Surface EMG based muscle fatigue evaluationin biomechanics. Clinical Biomechanics, 24, 327-340.

Clark, B., \& Taylor, J. (2011). Age-related changes in motor cortical properties and voluntary activation of skeletal muscle. Curr Aging Sci , 3 (4), 192199.

Contessa, P., Adam, A., \& De Luca, C. (2009). Motor unit control and force fluctuation during fatigue. Journal of Applied Physiology , 107 (1), 235243. 
Cook, D., O'Connor, P., Lange, G., \& Steffener, J. (2007). Functional neuroimaging correlates of mental fatigue induced by cognition among chronic fatigue syndrome patients. Neurolmage, 36 (1), 108-122.

Cooper, J., Worthy, D., Gorlick, M., \& Maddox, W. (2013). Scaffolding Across the Lifespan in History-Dependent Decision Making. Psychol Aging, 28 (2), 505-514.

Fairclough, S., \& Houston, K. (2004). A metabolic mearure of mental effort. Biological Psychology , 66, 77-190.

Finneran, A., \& O'Sullivan, L. (2013). Effects of grip type and wrist posture on forearm EMG activity, endurance time and movement accuracy. International Journal of Industrial Ergonomics , 43 (1), 91-99.

Holtzer, R., Shuman, M., Mahoney, J., Lipton, R., \& Verghese, J. (2011). Cognitive fatigue defined in the context of attention network. Neuropsychol Dev Cogn B Aging Neuropsychol Cogn, 18 (1), 108-128.

Hoozemans, M., \& van Dieën, J. (2005). Prediction of handgrip forces using surface EMG of forearm muscles. Journal of electromyography and kinesiology, 15 (4), 358-366.

Hoshi, Y., Kobayashi, N., \& Tamura, M. (2001). Interpretation of near-infrared spectroscopy signals: a study with a newly developed perfused rat brain model. Journal of Applied Physiology, 90 (5), 1657-1662. 
Huang, C.M., Polk, T., Goh, J., \& Park, D. (2012). Both left and right posterior parietal activations contribute to compensatory processes in normal aging. Neuropsychologia, 50 (1), 55-66.

Insel, K., Morrow, D., Brewer, B., \& Figueredo, A. (2006). Executive function, working memory, and medication adherence among older adults. Journal of Gerontology, 6 (2), 102-107.

Klaassen, F., Evers, F., Gront, R., de Backes, W., Veltman, D., \& Jolles, J. (2014). Working memory in middle-aged males: Age-related brain activation changes and cognitive fatigue effects. Biological Psychology, 96, 134-143.

Lin, C., Chow, L., Wei, S., Lieu, F., Chiang, S., \& Sung, W. (2014). Influence of aging on bimanual coordination control. Experimental Gerontology, 53 (40-47).

Liu, J., Lewandowski, B., Karakasis, C., Yao, B., Sieminow, V., Sahgal, V., et al. (2007). Shifting of activation center in the brain during muscle fatigue: an explanation of minimal central fatigue? Neurolmage, 35 (1), 299-307.

Liu, J., Shan, Z., Zhang, L., Sahgal, V., Brown, R., \& Yue, G. (2003). Human Brain Activation During Sustained and Intermittent Submaximal Fatigue Muscle Contractions: An fMRI Study. Journal of Neurophysiology, 90 (1), 300-312.

Lorist, M., Boksem, M., \& Ridderinkhof, K. (2005). Impaired cognitive control and reduced cingulate activity during mental fatigue. Cognitive Brain 
Research, 24, 199-205.

Madeleine, P., Bajaj, P., Sogaard, K., \& Arendt-Nielsen, L. (2001).

Mechanomyography and electromyography force relationships during concentric, isometric and eccentric contraction. Journal of Electromyography and Kinesiology , 11, 113-121.

Marcora, S., Staiano, W., \& Manning, V. (2009). Mental fatigue impairs physical performance in humans. J Appl Physiol , 106, 857-864.

Mehta, R. K. (In press). Impacts of obesity and stress on neuromuscular fatigue developement and associated heart rate variability. International Journal of Obesity.

Mehta, R., \& Agnew, M. (2011). Effects of concurrent physical and mental demands for a short duration static task. International Journal of Industrial Ergonomics, 41 (488-493).

Mehta, R., \& Agnew, M. (2013). Exertion-dependent effects of physical and mental workload on physiological outcomes and task performance. IIE Transactions on Occupational Ergonomics and Human Factors, 1, 3-15.

Mehta, R., \& Agnew, M. (2012). Influence of mental workload on muscle endurance, fatigue, and recovery during intermittent static work. European Journal of Applied Physiology, 112, 2891-2902.

Mehta, R., \& Parasuraman, R. (2014). Effects of mental fatigue on the developement of physical fatigue: a neuroergonomic approach. Human Factors: The Journal of the Human Factors and Ergonomics Society , 56 
(4), 645-656.

Milham, M., Erickson, K., Banich, M., Kramer, A., Webb, A., Wszalek, T., et al. (2002). Attentional control in the aging brain: insights from an fMRI Study of the Stroop Task. . Brain and Cognition , 49, 277-296.

Noble, J., Eng, J., Kokotilo, K., \& Boyd, L. (2011). Aging effects on the control of grip force magnitude: An fMRI study . Experimental Gerontology, 46, 453461.

Pagani, M., Mazzuero, G., Ferrari, A., \& et al. (1991). Sympathovagal interaction during mental stree. Circulation, 83, 43-51.

Park, D., \& Reuter-Lornez, P. (2009). The Adaptive Brain: Aging and Neurocognitive Scaffolding. Annu Rev Psychol , 60, 173-96.

Pereira, H., Spears, V., Schlinder-Delap, B., Yoon, T., Harkins, A., Nielson, K., et al. (2015). Sex Differences in Arm Muscle Fatigability With Cognitive Demand in Older Adults. Clinical Orthopaedics and Related Research, 110.

Pereira, H., Spears, V., Schlinder-Delap, B., Yoon, T., Nielson, K., \& Hunter, S. (2015). Age and sex differences in steadiness of elbow flexor muscles with imposed cognitive demand. European journal of applied physiology, $1-13$

Power, G., Dalton, B., \& Rice, C. (2013). Human neuromuscular structure and function in old age: A brief review. Journal of Sport and Health Science, 2, 215-226. 
Qin, S., Hermans, E., van Marle, H., Luo, J., \& Fernandez, G. (2009). Acute psychological stress reduces working memory-related activity in the dorsolateral prefrontal cortex. Biol Psychiatry, 66, 25-32.

Ranganatham, V., Siemionow, V., Sahgal, V., \& Yue, G. (2001). Effects of Aging on Hand Function. Journal of the American Geriatrics Society , 49 (11), 1478-1484.

Rantanen, T., Guralnik, J., Sakari-Rantala, R., Leveille, S., Simonsick, E., Link, S., et al. (1999). Disability, physical activity, and muscle strength in older women: the women's healthy and aging study. Arch Phys Med Behavil, $80,130-135$.

Raz, N., Lindenberger, U., Rodrigue, K., Kennedy, K., Head, D., Williamson, A., et al. (2005). Regional brain changes in aging healthy adults: general trends, individual differences and modifiers. Cereb Cortex, 15 (11), 16761689.

Rypma, B., \& D'Esposito, M. (2000). Isolating the neural mechanisms of agerelated changes in human working memory. Nature Neuroscience, 3 (5), 509-515.

Shibuya, K., \& Kubnyama, N. (2007). Human motor cortex oxygenation during exhaustive pinching task. Brain Research, 1156, 120-124.

Shortz, A., Van Dyke, S., \& Mehta, R. (2012). Neural Correlates of Physical and Mental Fatigue. Proceedings of the Human Factors and Ergonomics Society Annual Meeting, 56, 2172-2176. 
Smets, E. M., Garssen, B., Bonke, B., \& De Haes, J. (1995). The multidimensional fatigue inventory (MFI) psychometric qualities of an instrument to assess fatigue. Journal of Psychosomatic Research, 39, 315-325.

Takahara, D., Inoue, K.-i., Hirata, Y., Miyachi, S., Nambu, A., Takada, M., et al. (2012). Multisynaptic projections form the ventrolateral prefontal cortex to the dorsal premotor cortex in macaques. European Journal of Neuroscience , 36, 3365-3375.

Thayer, J., Ahs, F., Fredrikson, M., Sollers III, J., \& Wager, T. (2012). A metaanalysis of heart rate variability and neuroimaging studies: implications for heart rate variability as a marker of stress and health. Neuroscience and Biobehavioral Reviews, 36, 747-756.

Vøllestad, N. K. (1997). Measurement of human muscle fatigue. Journal of Neuroscience Methods , 74, 219-227.

Wang, M., Gamo, N., Jin, L., Wang, X., Laubach, M., Mazer, J., et al. (2011). Neuronal basis of age-related working memory decline. Nature, 476, 747-756.

Wood, R., Maraj, B., Lee, M., \& Reyes, R. (2002). Short-term heart rate variability during a cognitive challeng in young and older adults. Age and Ageing, 31, 131-135. 
Wood, R., Wood, W., Welsch, M., \& Avenal, P. (1998). Physical activity, mental stress, and short-term heart rate varability in patients with ischemic heart disease. J Cardiopul Rehab, 18, 271-276.

Yassierli, Nussbaum, M., Iridiastadi, H., \& Wojcik, L. (2007). The influence of age on isometric endurance and fatigue is muscle dependent: a study of shoulder abduction and torso extension. Ergonomics , 50 (1), 26-45.

Yoon, T., Schlinder, B., Griffith, F., \& Hunter, S. (2008). Age-related muscle fatigue after a low-force fatiguing contraction is explained by central fatigue. Muscle \& Nerve , 37, 457-466. 